\title{
Erratum to: Postural change in volunteers: sympathetic tone determines microvascular response to cardiac preload and output increases
}

\author{
Eva Klijn $^{1} \cdot$ Sjoerd Niehof $^{2}$ - A. B. Johan Groeneveld ${ }^{1} \cdot$ Alexandre Pinto Lima $^{1}$ • \\ Jan Bakker ${ }^{1} \cdot$ Jasper van Bommel $^{1}$
}

Published online: 26 October 2015

(c) Springer-Verlag Berlin Heidelberg 2015

\section{Erratum to: Clin Auton Res DOI 10.1007/s10286-015-0286-x}

Unfortunately, the last sentence of abstract's result section was omitted during publication process. The complete corrected abstract is given below.

\begin{abstract}
Purpose Microvascular perfusion may be a non-invasive indicator of fluid responsiveness. We aimed to investigate which of the microvascular perfusion parameters truly reflects fluid responsiveness independent of sympathetic reflexes.

Methods Fifteen healthy volunteers underwent a postural change from head up tilt (HUT) to the supine position, diminishing sympathetic tone, followed by a $30^{\circ}$ passive leg raising (PLR) with unaltered tone. Prior to and after the postural changes, stroke volume (SV) and cardiac output (CO) were measured, as well as sublingual microcirculatory perfusion (sidestream dark field imaging), skin perfusion, and oxygenation (laser Doppler flowmetry and reflectance spectroscopy).
\end{abstract}

The online version of the original article can be found under doi:10.1007/s10286-015-0286-x.

Eva Klijn

evaklijn@hotmail.com

1 Department of Intensive Care, Erasmus MC University

Medical Center, PO box 2040, Room H 621,

3000 CA Rotterdam, The Netherlands

2 Department of Anesthesiology, Erasmus MC University Medical Center, Rotterdam, The Netherlands
Results In responders (subjects with $>10 \%$ increase in $\mathrm{CO}$ ), the HUT to supine change increased $\mathrm{CO}, \mathrm{SV}$, and pulse pressure, while heart rate, systemic vascular resistance, and mean arterial pressure decreased. Additionally, microvascular flow index, laser Doppler flow, and microvascular hemoglobin oxygen saturation and concentration also increased. During PLR, only CO, SV and sublingual functional capillary density increased in responders ( $>5 \%$ increase in $\mathrm{CO}$ ), whereas systemic vascular resistance decreased less than from HUT to supine. Conclusion When preload and forward flow increase in association with a decrease in sympathetic activity, microvascular blood flow increases in the skin and in the sublingual area. When preload and forward flow increase with little to no change in sympathetic activity, only sublingual functional capillary density increases. Therefore, our results indicate that sublingual functional capillary density is the best parameter to use when evaluating fluid responsiveness independent of changes in sympathetic tone. 